\section{Upper and lower bounds on a system's bandwidth based on its zero-value time constants}

B. Hong ${ }^{凶}$ and A. Hajimiri

It is shown that for systems with no zeros and no complex poles, the classical estimate of the $3 \mathrm{~dB}$ cutoff frequency based on the sum of the zero-value time constants (ZVTs) is always conservative. The opposite problem is also solved, whereby a non-trivial upper bound on the cutoff frequency which depends only on the sum of the ZVTs and the system's order is derived. It is demonstrated that both bounds are tight - specifically, the lower bound is approached by making one of the system's poles increasingly dominant, whereas the best possible bandwidth is achieved when all of the system's poles overlap. The impact of complex poles on the results is also discussed.

Introduction: The estimate of a low-pass circuit's $3 \mathrm{~dB}$ bandwidth via the method of zero-value time constants (ZVTs) is well-known [1-3]. This procedure entails computing the time constant of each reactive element in a circuit based on the resistance it sees when all other reactive elements are zero-valued (capacitors opened, inductors shorted). The inverse of the sum of these ZVTs is then taken to be an estimate of the circuit's $3 \mathrm{~dB}$ high-cutoff frequency. For a linear time-invariant $n$ th-order system with $m$ zeros and $n$ poles $(m<n)$ whose transfer function can be written as

$$
H(s)=\frac{a_{0}+a_{1} s+a_{2} s^{2}+\cdots+a_{m} s^{m}}{1+b_{1} s+b_{2} s^{2}+\cdots+b_{n} s^{n}},
$$

it can be shown [1-3] that the sum of the ZVTs is equal to $b_{1}$. For now, we will consider systems with no zeros $\left(a_{1}=a_{2}=\cdots=a_{m}=0\right)$, which serve as a good model for circuits where the zeros occur at very high frequencies beyond the passband and are hence unimportant. We thus rewrite the transfer function as

$$
H(s)=\frac{H_{0}}{1+b_{1} s+b_{2} s^{2}+\cdots+b_{n} s^{n}},
$$

where $\left|H_{0}\right|=\left|a_{0}\right|$ is the DC gain of the system. We then argue that as the frequency increases from DC, the first term in the denominator that becomes significant is $b_{1} s$, and so near the $-3 \mathrm{~dB}$ point the system can be approximated as

$$
H(s) \approx \frac{H_{0}}{1+b_{1} s}
$$

from which the ZVT bandwidth estimate of $\omega_{c} \approx 1 / b_{1}$ follows [1, 3]

In this Letter, we prove that for a system whose poles are all real, this ZVT estimate of the bandwidth is always a lower bound on the system's actual bandwidth $\omega_{c}$. We also prove a non-trivial upper bound on $\omega_{c}$ that depends only on the sum of the ZVTs $b_{1}$ and the system's order $n$.

\section{Statement of the theorem:}

Consider a linear time-invariant nth-order low-pass system with no zeros and no complex poles whose transfer function is

$$
H(s)=\frac{H_{0}}{1+b_{1} s+b_{2} s^{2}+\cdots+b_{n} s^{n}} .
$$

The system's $3 d B$ bandwidth, or high-cutoff frequency, is defined as the (lowest) angular frequency $\omega_{c}$ that satisfies

$$
\left|H\left(j \omega_{c}\right)\right| \equiv \frac{\left|H_{0}\right|}{\sqrt{2}} .
$$

If $n=1$, then trivially $\omega_{c}=1 / b_{1}$. For $n>1$, the following lower and upper bounds on $\omega_{c}$ hold:

$$
\frac{1}{b_{1}}<\omega_{c} \leq \frac{n}{b_{1}} \sqrt{2^{1 / n}-1}
$$

Furthermore, these inequalities are tight, or achievable, in the sense that there exists systems whose bandwidths are arbitrarily close to the lower bound or are given by the upper bound.
A couple of comments are in order:

1) The lower bound also holds for systems with complex poles whose quality factors are no larger than $Q \leq \sqrt{2}$. (The upper bound does not.) We will prove this separately in a later section.

2) The lower bound is physically intuitive. Loosely speaking, the ZVT estimate $1 / b_{1}$ considers the worst case scenario where the system's reactive elements 'energise' one after the other, as the time constants are added together. In general, however, the system's energy-storage elements may energise in parallel, leading to a 'faster' response than that dictated by $b_{1}$.

Proof of the lower bound: Assume $n>1$. Since all the poles are real, by the fundamental theorem of algebra, the denominator of (4) can be factored as

$$
H(s)=H_{0} \prod_{i=1}^{n} \frac{1}{1+\tau_{i} s},
$$

where $\tau_{i} \in \mathbb{R}_{++}$for $i=1, \ldots, n$ are the time constants associated with the system's $n$ poles. (Note that the $\tau_{i}$ 's are equal to the system's ZVTs only if the poles are decoupled from one another [3]. In general, this is not the case. It is only true that the ZVTs and the $\tau_{i}$ 's have the same total sum, namely $b_{1}$.) To simplify the notation, we define the following variables for $i=1, \ldots, n$ :

$$
x_{i}:=\omega \tau_{i},
$$

where the frequency $\omega$ will be specified based on context. Lastly, based on the easily seen fact that $b_{1}=\sum_{i=1}^{n} \tau_{i}$, we can also write $\omega b_{1}=\sum_{i=1}^{n} x_{i}$.

To make the proof more analytically tractable, we reformulate the problem statement in an equivalent way. By definition of the bandwidth, the lower bound $\omega_{c}>1 / b_{1}$ is equivalent to:

$$
|H(j \omega)|>\frac{\left|H_{0}\right|}{\sqrt{2}}
$$

for all $\omega \leq 1 / b_{1}$. That is, the system's magnitude is always above $-3 \mathrm{~dB}$ (relative to the DC gain) for frequencies up to $1 / b_{1}$, the ZVT estimate of the high-cutoff frequency. We can then express the problem statement as follows: for $x_{i}>0 \forall i$, show that

$$
\begin{gathered}
\prod_{i=1}^{n}\left(1+x_{i}{ }^{2}\right)<2 \\
\text { subject to } \sum_{i=1}^{n} x_{i} \leq 1 .
\end{gathered}
$$

Now, we proceed with the proof proper. First, observe the following simple fact: given constants $a$ and $b$ such that $0<a, b<1$, it holds true that

$$
\begin{aligned}
\left(1+a^{2}\right)\left(1+b^{2}\right) & =(1-a b)^{2}+(a+b)^{2} \\
& <1+(a+b)^{2},
\end{aligned}
$$

where equality is approached by taking $a \rightarrow 0$ or $b \rightarrow 0$ (or both). We need to repeat this argument for a total of $n-1$ times, where the $k$ th step features $a=\sum_{i=1}^{k} x_{i}$ and $b=x_{k+1}$. For each step, it is apparent that $0<a, b<1$, since $x_{i}>0 \forall i$ combined with the constraint (11) implies that $\sum_{i \in S} x_{i}<1 \forall S \subset\{1, \ldots, n\}$. Thus, we arrive at

$$
\prod_{i=1}^{n}\left(1+x_{i}{ }^{2}\right)<1+\left(\sum_{i=1}^{n} x_{i}\right)^{2} \leq 2,
$$

where the final inequality follows from the constraint $\sum_{i=1}^{n} x_{i} \leq 1$. By letting one of the $x_{i}$ 's approach unity (which takes the remaining $x_{i}$ 's to zero), we can also see that this inequality is tight. This establishes the lower bound.

Notice what is happening here physically. We are 'merging' the system's poles together one at a time (by adding their time constants together), and with each step, the bandwidth of the system worsens until we end up with a first-order system whose bandwidth is exactly equal to the ZVT estimate $1 / b_{1}$. The proof also shows how this lower bound is achievable: as one of the system's poles becomes increasingly dominant (where to dominate means to have a larger time constant), the system's bandwidth will approach the lower bound dictated by (6). 
Proof of the upper bound: The proof of the upper bound is somewhat similar in spirit, but we take a slightly different approach. Consider the following system:

$$
H_{\max }(s):=\frac{H_{0}}{(1+\bar{\tau} s)^{n}},
$$

where

$$
\bar{\tau}:=\frac{1}{n} \sum_{i=1}^{n} \tau_{i}=\frac{b_{1}}{n}
$$

is the arithmetic mean of all the time constants. Notice that this system has the same $b_{1}$ coefficient as (4). We claim that of all $n$ th-order systems (with no zeros or complex poles) that share the same $b_{1}$ coefficient, $H_{\max }(s)$ has the best, or maximum, bandwidth. In other words, for a given sum of the ZVTs, the best bandwidth is achieved by stacking all of the poles on top of each other. Appealing to the fact that $\left|H_{\max }(j \omega)\right|$ is monotonically decreasing with $\omega$, we can establish this claim by proving that the magnitude of $H_{\max }$ at the cutoff frequency $\omega_{c}$ of (4) is always no less than $-3 \mathrm{~dB}$ (relative to the $\mathrm{DC}$ gain):

$$
\left|H_{\max }\left(j \omega_{c}\right)\right| \geq\left|H\left(j \omega_{c}\right)\right| \equiv \frac{\left|H_{0}\right|}{\sqrt{2}} .
$$

Letting $\omega=\omega_{c}$ in (8), this is equivalent to the following: given $x_{i}>0 \forall i$, show that

$$
\begin{gathered}
\left(1+\bar{x}^{2}\right)^{n} \leq 2 \\
\text { subject to } \prod_{i=1}^{n}\left(1+{x_{i}}^{2}\right)=2,
\end{gathered}
$$

where $\bar{x}:=\omega_{c} \bar{\tau} \equiv \sum_{i=1}^{n} x_{i} / n$ is the arithmetic mean of the $x_{i}$ 's.

The general idea of the proof is to 'move' the $\tau_{i}$ 's to $\bar{\tau}$ one by one, whilst improving the bandwidth of the system with each move. To that end, we will actually prove, subject to (18), the equivalent inequality

$$
\prod_{i=1}^{n}\left(1+x_{i}^{2}\right) \geq\left(1+\bar{x}^{2}\right)^{n} .
$$

We now proceed with the proof proper. If $x_{i}=\bar{x} \forall i$, the upper bound is trivially attained, so assume otherwise. Then, $\exists x_{p}>\bar{x}, x_{q}<\bar{x}$. Denote $\Delta_{p}:=x_{p}-\bar{x}>0$. We will now establish the following inequality:

$$
\left(1+x_{p}{ }^{2}\right)\left(1+x_{q}{ }^{2}\right)>\left(1+\bar{x}^{2}\right)\left[1+\left(x_{q}+\Delta_{p}\right)^{2}\right] .
$$

To see this, define the function

$$
f(\zeta):=\left[1+\left(x_{p}-\zeta\right)^{2}\right]\left[1+\left(x_{q}+\zeta\right)^{2}\right] .
$$

We want to show that $f(0)>f\left(\Delta_{p}\right)$. One can compute that

$$
f(0)-f\left(\Delta_{p}\right)=\Delta_{p}\left(\bar{x}-x_{q}\right)\left\{\Delta_{p}\left(\bar{x}-x_{q}\right)+2\left[1-\bar{x}\left(x_{q}+\Delta_{p}\right)\right]\right\} .
$$

By construction, $x_{q}<\bar{x}$. Hence, to establish the strict positivity of $f(0)-f\left(\Delta_{p}\right)$, we need only check that $1-\bar{x}\left(x_{q}+\Delta_{p}\right)>0$. Since the $x_{i}$ 's are positive, by the constraint (18), we know that $x_{i}<1 \forall i$. Then, we can see that $0<\bar{x}<x_{p}<1$ and $0<x_{q}+\Delta_{p}<\bar{x}+\Delta_{p}=x_{p}<1$. Thus, $0<\bar{x}\left(x_{q}+\Delta_{p}\right)<1$, which shows that $f(0)>f\left(\Delta_{p}\right)$. This proves (20).

By renaming $x_{p} \leftarrow \bar{x}$ and $x_{q} \leftarrow x_{q}+\Delta_{p}$, we have effectively moved $x_{p}$ to the mean $\bar{x}$ while moving $x_{q}$ (which was on the other side of $\bar{x}$ ) by the same but opposite amount in order to maintain the average $\bar{x}$. This procedure, as we just showed, decreases $\prod_{i=1}^{n}\left(1+x_{i}{ }^{2}\right)$. We now repeat the above algorithm until all of the $x_{i}$ 's are equal to $\bar{x}$, which yields the right-hand-side of (19), proves (17) and thus also (16), and finally establishes our claim.

Now that we have shown that $H_{\max }(s)$ is indeed the $n$ th-order system (with no zeros or complex poles) with the best bandwidth for the given $b_{1}$ coefficient, all that is left for us to do is to compute this optimal bandwidth. It is a simple exercise to show that the bandwidth of $H_{\max }(s)$, which we shall denote by $\omega_{\max }$, is given by

$$
\omega_{\max }=\frac{n}{b_{1}} \sqrt{2^{1 / n}-1} .
$$

This proves the upper bound and also establishes its tightness.

There is a simple mathematical intuition that we can glean from the upper bound. Since the lower bound is approached when one of the poles is dominant - in the extreme case all non-dominant poles are infinitely far away and we are left with the first-order system $H_{0} /\left(1+b_{1} s\right)$ whose bandwidth is exactly $1 / b_{1}-$ it makes sense that the upper bound is attained when none of the poles dominates, in which case all the poles are identical.
Impact of complex poles: Here, we show that the lower bound $\omega_{c}>1 / b_{1}$ of (6) still holds when the system has complex poles whose quality factors do not exceed $Q \leq \sqrt{2}$. Consider the canonical form of the denominator polynomial that describes a pair of complex conjugate poles

$$
D(s)=1+\frac{s}{Q \omega_{0}}+\left(\frac{s}{\omega_{0}}\right)^{2},
$$

where $Q>1 / 2$. The time constant associated with this complex pole pair is the coefficient of the $s$ term $\tau=1 /\left(Q \omega_{0}\right)$, and so we proceed to denote, just like before, $y:=\omega \tau=\omega /\left(Q \omega_{0}\right)$. Then, it holds true that

$$
|D(j \omega)|^{2}=\left[1-(Q y)^{2}\right]^{2}+y^{2}<1+y^{2}
$$

if $0<y<1$ and $Q \leq \sqrt{2}$. Now, to account for $m$ pairs of complex conjugate poles in the original system, the left-hand-side of (10) is multiplied by the additional term $\prod_{j=1}^{m}\left(\left[1-\left(Q_{j} y_{j}\right)^{2}\right]^{2}+y_{j}^{2}\right)$ and the constraint (11) is modified to $\sum_{i=1}^{n} x_{i}+\sum_{j=1}^{m} y_{j} \leq 1$. Noting that $0<y_{j}<1 \forall j$ due to the modified constraint and applying the above reasoning of (24) to first deal with the complex poles, we can subsequently proceed with the proof of the lower bound as usual.

It is left as a tedious algebra exercise to show that the upper bound does not hold when the system has complex poles. More precisely, for any pair of complex conjugate poles, there exists a system with those poles whose bandwidth exceeds the upper bound of (6).

Conclusion: In this Letter, we proved that a (strict) lower bound on the bandwidth of a low-pass system with no zeros, order exceeding unity, and whose complex poles feature quality factors satisfying $Q \leq \sqrt{2}$ is given by its well-known ZVT estimate

$$
\omega_{c}>\frac{1}{b_{1}}
$$

and this bound can be approached by making one of the system's (real) poles increasingly dominant. We also proved that an upper bound on the bandwidth of an $n$ th-order low-pass system with no zeros and no complex poles is given by

$$
\omega_{c} \leq \frac{n}{b_{1}} \sqrt{2^{1 / n}-1}
$$

and this bound is attained when all the poles are at the same location. Both bounds are equally important from a conceptual standpoint, as the lower bound should not be 'favoured' over the upper bound, except perhaps for the reason that the system performs at least as well as the lower bound - i.e. the lower bound serves as a conservative estimate. However, in a system where all the poles are around the same ballpark of frequencies, the upper bound may actually serve as a better estimate of the bandwidth.

Additionally, to crudely account for the effect that zeros have on the bandwidth, one can replace the ZVTs with the modified ZVTs described in [3], whose sum is equal to $b_{1}-a_{1} / a_{0}$ from (1). However, with this substitution for $b_{1}$, the rigor of the bounds of (6) no longer prevails.

In passing, we would also like to mention that the low-cutoff $\omega_{c, l}$ of an $n$ th-order (where $n>1$ ) high-pass system with no non-DC zeros and equivalent assumptions on complex poles satisfies the following analogous bounds:

$$
\frac{1}{n \sqrt{2^{1 / n}-1}} \leq \frac{\omega_{c, l}}{\sum_{k} 1 / \tau_{k}^{\infty}}<1
$$

where the $\tau_{k}^{\infty}$ 's are the system's infinite-value time constants.

Acknowledgments: The authors thank P. Khial, A. Taeb, K.-C. Chen, and A. Agarwal, all with Caltech, for their enlightening technical discussions.

B. Hong and A. Hajimiri (Department of Electrical Engineering, California Institute of Technology, Pasadena, CA, USA)

$\bowtie$ Corresponding E-mail: bhong@caltech.edu

\section{References}

1 Thornton, R.D., Searle, C.L., Pederson, D.O., Adler, R.B., and Angelo, E.J.: 'Multistage transistor circuits' (Wiley, New York, 1965)

2 Cochran, B.L., and Grabel, A.: 'A method for the determination of the transfer function of electronic circuits', Trans. Circuit Theory, 1973, 20, (1), pp. 16-20

3 Hajimiri, A.: 'Generalized time- and transfer-constant circuit analysis', Trans. Circuits Syst. I, Regul. Pap., 2010, 57, (6), pp. 1105-1121 